(C) 1983. The Genetical Society of Great Britain

\title{
DYNAMICS OF THE $B$ CHROMOSOME POLYMORPHISM IN RYE II. ESTIMATES OF PARAMETERS
}

\author{
R. B. MATTHEWS AND R. N. JONES \\ Department of Agricultural Botany, University College of Wales, Aberysturth, U.K.
} Received 7.vii.82

\section{SUMMARY}

The computer simulation model presented in the first paper in this series identified six parameters which were considered to represent the main processes determining the transmission rates and distribution characteristics of the $B$ chromosomes of rye. Basically it was found that a drive mechanism is provided by the directed nondisjunction of $B$ s at first pollen grain, and first egg cell, mitosis and that, the other parameters are largely concerned in modifying the drive and in regulating the final equilibrium $B$ levels.

The $B$ s were found to be "selfish" and to be capable of maintaining and dispersing themselves throughout populations even in the face of negative maladaptive effects upon reproductive and physiological fitness.

In this paper estimates are presented, from published sources, of the real values of the parameters concerned. These estimates have been used to assess the application of the model and to determine the range and means of the parameters in relation to differing genetic and environmental influences.

\section{INTRODUCTION}

In the first paper in this series we presented a computer model simulating the dynamics of the transmission and distribution characteristics of the $B$ chromosomes of rye (Matthews and Jones, 1982). The model was based on a mathematical interpretation, and representation, of certain parameters which determine the heredity and accumulation of $B$ chromosomes in random mating populations. The parameters determining equilibrium frequencies were identified as follows: $\beta$, the rate of nonpairing of $B$ s at meiosis; $\lambda$, the rate of loss of nonpaired $B s ; \mu$, rate of nondisjunction at first pollen grain and first egg cell mitosis; $\alpha$, direction rate (into generative or vegetative nucleus) of nondisjoined $B s$; gametic weighting factors for relative competitive abilities of different $B$-classes of pollen, and hence gametes, and plant weighting factors to account for adverse developmental effects which $B$ chromosomes have upon the sporophyte phase of the life cycle.

In applying the model account was taken of the fact that plants with four or more $B$ s are virtually sterile, and the simulations all commenced with an arbitrary and initial low frequency of $0.01 \mathrm{~B} /$ plant. Parameters were varied over the range of values $0 \cdot 0-1 \cdot 0$, in steps of $0 \cdot 1$, either individually or in various combinations, and an understanding was gained of the relative contribution made by each of the parameters to the final $B$ frequency equilibrium levels within populations. Essentially it was concluded that the directed nondisjunction constituted a powerful drive mechanism for the accumulation and distribution of the $B \mathrm{~s}$, and that this mechanism alone, aside from any involvement of adaptation and natural 
selection on the basis of phenotype, is sufficient to account for the widespread polymorphism of the $B$ s of rye in natural populations. Equilibrium frequencies depend upon the rate, and direction, of nondisjunction, and the extent to which this tendency to accumulate by mitotic drive in the gametophyte is offset by meiotic elimination and by reducing physiological and competitive fitness of the $B$-carrying gametophyte and sporophyte stages.

The model was developed on a theoretical basis and did not take into account the real values of the parameters concerned. In this paper we present estimates of these real values, based on published sources, from studies on the inheritance of the $B$ s in different experimental strains of rye collected from a number of wild populations. There is evidence for wide variation in the parameter values, relative to material of diverse origin and habitat, and evidence too from using these estimates in the model that the real values of the parameters are such as to produce an accumulating situation leading to equilibrium $B$-frequencies in populations.

\section{METHODS}

Estimates of parameter values and their standard errors were made by the method of maximum likelihood, as described and discussed by Mather $(1963,1972)$. It is the most efficient way of estimating parameters in models. It reduces essentially to the method of least squares when the observations are normally distributed, but does not depend on normality. It is therefore more general than least squares and in this case is applied to data with a binomial distribution.

By observing the behaviour of $B$ chromosomes at meiosis, and their distribution in the microspores afterwards (metaphase of first pollen grain mitosis), it is possible using the method of maximum likelihood to estimate both nonpairing rate $\beta$ and the nonpaired loss rate parameter $\lambda$. Similarly, by observation of the first pollen division itself, estimates of the nondisjunction rate $\mu$ and direction rate $\alpha$ can be made. The distribution of different $B$ classes of plants among progeny of controlled crosses can also be used to give estimates of $\mu$ and $\alpha$ in those cases where the $\beta$ and $\lambda$ values are already known. A small amount of data is also presented from which estimates of the gametic weighting factors and the plant weighting factors are made.

\section{Results}

(i) Estimates of parameters based on cytological data

(a) Hasegawa (1934) studied mitosis in $1 B$ rye pollen grains and was the first to see the nondisjunction process occurring. He presented data showing the distribution pattern of daughter $B$ chromosomes following first pollen grain mitosis, as summarised in table 1 . From these data it can be calculated that in thirteen, out of a total of 21 , anaphases the generative nucleus received $2 B \mathrm{~s}$, in two it received $1 B$ and in six it received none at all. In addition 6 out of 138 telophases, or 4.35 per cent of cells, showed loss of both $B$ s due to lagging. In a total of 21 anaphases therefore one would expect 


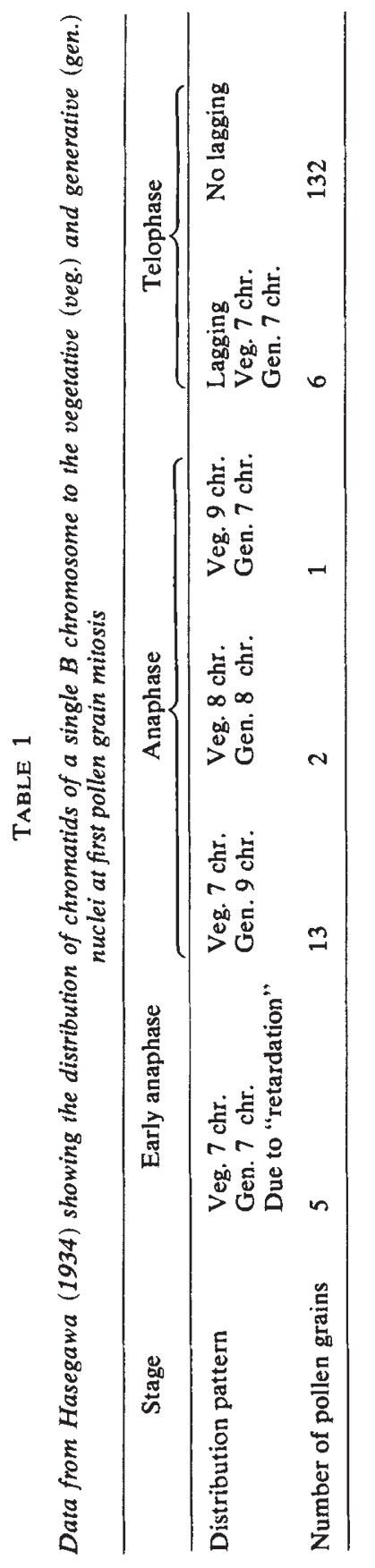


$21 \times 4.35$ per cent, or almost one more anaphase pollen grain, with the generative nucleus receiving no $B$ s. This can be summarised with regard to the generative nucleus as follows:

$$
0 B: 7(0.318), \quad 1 B: 2(0.091), \quad 2 B: 13(0.591) \text {. }
$$

Using the equations derived in the previous paper for $1 B$ microspores, and equating them to these values, it is possible to solve for $\mu$ and $\alpha$ :

$$
\begin{aligned}
& 0 B: \mu(1-\alpha)=0.318 \\
& 1 B: 1-\mu=0.091 \\
& 2 B: \mu \alpha=0.591 .
\end{aligned}
$$

This gives values of $\mu=0.909$ and $\alpha=0.645$, indicating that nondisjunction has occurred in 91 per cent of the cells and that in 65 per cent of these the $B$ s have entered the generative nucleus. Of those not entering the generative nucleus, it seems that the larger proportion i.e., 6 out of 7 (86 per cent) are due to loss from both nuclei, while only 1 out of 7 (14 per cent) are due to nondisjoined chromatids entering the vegetative nucleus instead.

(b) Müntzing (1946) observed metaphase of the first pollen grain division and recorded the distribution of the daughter $B$ chromosomes in both $2 B$ and $4 B$ plants of the variety Östgöta Gråråg. By using the maximum likelihood method, the best values of the nonpairing rate $\beta$ and the nonpaired loss rate $\lambda$ were found (table 2(a)). Further data are presented in the same paper showing the distribution of $B$ s in the progeny of a $0 B \times 2 B$ cross (table 2(b)). Also shown in this table are the values that would be expected assuming that every $B$ chromosome present in the pollen grains underwent directed nondisjunction. It can be seen that there are fewer $B$ s than

TABLE 2

Estimates of parameters based on data for the Swedish rye variety Östgöta Gråråg, given in Müntzing, 1946

(a) Nonpairing rate ( $\beta)$ and nonpaired loss rate $(\lambda)$, from observations on first pollen grain mitosis

\begin{tabular}{crrrrrrr}
\hline \multirow{2}{*}{$\begin{array}{c}\text { B chromosome } \\
\text { class of plant }\end{array}$} & $0 B$ & $1 B$ & $2 B$ & $3 B$ & $4 B$ & $\beta$ & $\lambda$ \\
\hline $2 B$ & 62 & 100 & 10 & - & - & 0.708 & 0.427 \\
$4 B$ & 1 & 19 & 21 & 3 & 1 & 0.593 & 0.300 \\
\hline
\end{tabular}

(b) Nondisjunction rate $(\mu)$ and direction rate $(\alpha)$ estimated from crossing data

\begin{tabular}{lccccccc}
\hline Cross & \multicolumn{8}{c}{$B$-classes in progeny } & & & \\
$0 B \times 2 B$ & $0 B$ & $1 B$ & $2 B$ & $3 B$ & $4 B$ & $\mu$ & $\alpha$ \\
\hline $\begin{array}{l}\text { Observed nos. } \\
\text { Expected nos. } \\
\text { with complete } \\
\text { nondisjunction }\end{array}$ & 69 & 19 & 62 & - & 4 & 0.806 & 0.852 \\
\hline
\end{tabular}


might be expected, indicating that complete directed nondisjunction is not occurring. Estimates made using the maximum likelihood method confirm this although these values may be lower than the actual rates, as no account can be made of loss due to differential selection of gametes. Disturbances at the second pollen grain mitosis may also alter the distribution (Puertas et al., 1979). The value for $\alpha$ of 0.852 indicates that about 85 per cent of $B$ s are included in the generative nucleus. Müntzing further noted that in 209 telophases, 10 contained eliminated or lagging fragments. This represents 4.8 per cent. Thus of the 15 per cent of total $B$ s lost it seems as if $4 \cdot 8 / 15(=32$ per cent $)$ are lost from both nuclei, while 6 per cent are directed to the vegetative nucleus rather than to the generative nucleus. This contrasts markedly with the results of Hasegawa given above.

(c) Kishikawa's (1965) observations on meiosis in Japanese rye are summarised in table 3 . The data show the extent of pairing between $B$ chromosomes (table 3(a)), from which $\beta$ can be estimated, and the numbers of micronuclei formed (table $3(b)$ ), from which $\lambda$ can

TABLE 3

Estimates of parameters based on data for a Japanese rye variety, given in Kishikawa, 1965

(a) Estimates of nonpairing rate $(\beta)$ of $B$ s from observations on metaphase I of meiosis

\begin{tabular}{cccccccccc}
\hline \multirow{8}{*}{$\begin{array}{c}B \text { chromosome } \\
\text { class of plant }\end{array}$} & I & II & III & II + I & IV & III + I & 2II & II + 2I & $\beta$ \\
\hline $1 B$ & All & - & - & - & - & - & - & - & - \\
$2 B$ & 48 & 582 & - & - & - & - & - & - & $0 \cdot 076$ \\
$3 B$ & 2 & - & 268 & 130 & - & - & - & $\overline{7}$ & 0.005 \\
$4 B$ & 7 & - & - & - & 189 & 45 & 172 & 37 & 0.107 \\
\hline
\end{tabular}

(b) Estimates of nonpaired loss rate $(\lambda)$ based on frequency of micronuclei in microspore tetrads

\begin{tabular}{ccccc}
\hline $\begin{array}{c}B \text { chromosome } \\
\text { class of plant }\end{array}$ & $\boldsymbol{\beta}$ & Micronucleus freq. $(\boldsymbol{M})$ & \multicolumn{2}{c}{$\lambda$} \\
\hline $1 B$ & - & 0.200 & $2 M$ & 0.400 \\
$2 B$ & 0.076 & 0.006 & $M / \beta$ & 0.076 \\
$3 B$ & 0.005 & 0.017 & $2 M /(2 \beta+1)$ & 0.033 \\
$4 B$ & 0.107 & 0.020 & $M / 2 \beta$ & 0.094 \\
\hline
\end{tabular}

(c) Nondisjunction $(\mu)$ and direction rate $(\alpha)$ estimated from crossing data

\begin{tabular}{lccccccc}
\hline \multicolumn{1}{c}{} & \multicolumn{8}{c}{$B$-class frequencies } \\
in progeny & & & & \\
Crosses & $0 B$ & $1 B$ & $2 B$ & $3 B$ & $4 B$ & $\mu$ & $\alpha$ \\
\hline $1 B \times 1 B$ & 4 & 1 & 5 & 1 & 2 & 0.828 & 1.000 \\
$2 B \times 2 B(1962)$ & 3 & - & 36 & 6 & 17 & 0.947 & 0.657 \\
$2 B \times 2 B(1963)$ & 4 & 1 & 22 & 3 & 19 & 0.956 & 0.684 \\
$2 B \times 2 B$ (Total) & 7 & 1 & 58 & 9 & 36 & 0.951 & 0.669 \\
\hline
\end{tabular}


be estimated, once $\beta$ has been calculated. Kishikawa also performed various crosses and counted the progeny types, so from these data, and knowing values of $\beta$ and $\lambda$, it is possible to estimate $\mu$ and $\alpha$ (table 3(c)), if we assume no gametic selection is occurring. For the calculation of $\beta$ in $3 B$ and $4 B$ plants, the behaviour of trivalents is assumed to be the same as a bivalent and univalent and quadrivalents the same as two bivalents with regard to their distribution into the microspores, as the model assumes no greater association than bivalents. While this assumption is not strictly true, it was found that it gave close estimates of $\beta$, without the extra calculations needed to take separate trivalent and quadrivalent behaviour into account (Matthews, 1981). The $\lambda$ values were calculated from micronuclei frequencies according to the formulae shown in table 3 (b), the derivation of which is fully described in Matthews (1981). The $\mu$ and $\alpha$ values were calculated using the maximum likelihood method.

\section{TABLE 4}

Estimates of parameters in Japanese rye grown under experimentally contrasting temperature and soil moisture conditions. Data summarised from Kishikawa, 1970

(a) Influence of temperature on nonpairing $(\beta)$ and nonpaired loss rate $(\lambda)$

\begin{tabular}{|c|c|c|c|c|c|c|c|}
\hline \multirow[b]{2}{*}{ Treatment } & \multirow[b]{2}{*}{$B$-class } & \multicolumn{3}{|c|}{$\begin{array}{l}\text { Univalent classes } \\
\text { and frequency of cells }\end{array}$} & \multirow[b]{2}{*}{$\beta$} & \multirow{2}{*}{$\begin{array}{l}\text { Micronucleus } \\
\text { frequency }\end{array}$} & \multirow[b]{2}{*}{$\lambda$} \\
\hline & & 0 & 2 & 4 & & & \\
\hline Low & $2 B$ & 0.900 & $0 \cdot 100$ & - & $0 \cdot 100$ & 0.086 & 0.860 \\
\hline temp. & $4 B$ & 0.916 & 0.036 & 0.048 & 0.066 & 0.064 & 0.486 \\
\hline High & $2 B$ & 0.780 & 0.220 & - & 0.220 & $0 \cdot 168$ & 0.765 \\
\hline temp. & $4 B$ & 0.801 & 0.055 & 0.144 & $0 \cdot 172$ & 0.098 & 0.286 \\
\hline
\end{tabular}

(b) Influence of soil moisture content on nonpairing ( $\beta)$ and nonpaired loss rate $(\lambda)$

\begin{tabular}{lccccccc}
\hline \multicolumn{9}{c}{ Univalent classes } \\
Treatment & $B$-class & 0 & 2 & 4 & $\beta$ & frequency & $\lambda$ \\
\hline Moist & $2 B$ & 0.833 & 0.117 & - & 0.117 & 0.046 & 0.393 \\
Control & $2 B$ & 0.867 & 0.133 & - & 0.133 & 0.050 & 0.376 \\
Dry & $2 B$ & 0.850 & 0.150 & - & 0.150 & 0.071 & 0.467 \\
\hline
\end{tabular}

(c) Estimation of nondisjunction $(\mu)$ and direction rates $(\alpha)$ from progeny data of $2 B \times 2 B$ crosses

\begin{tabular}{lcccccccccc}
\hline & & \multicolumn{1}{c}{ Frequency in } \\
Treatment & Cross & $\beta$ & $\lambda$ & $0 B$ & $1 B$ & $2 B$ & $3 B$ & $4 B$ & $\mu$ & $\alpha$ \\
\hline Low temp. & $2 B \times 2 B$ & 0.100 & 0.860 & 7 & 4 & 43 & 15 & 80 & 0.925 & 0.863 \\
high temp. & $2 B \times 2 B$ & 0.220 & 0.765 & 9 & 10 & 62 & 17 & 52 & 0.877 & 0.831 \\
Moist & $2 B \times 2 B$ & 0.117 & 0.393 & 4 & 12 & 44 & 11 & 72 & 0.907 & 0.808 \\
Control & $2 B \times 2 B$ & 0.133 & 0.376 & 5 & 7 & 49 & 25 & 60 & 0.864 & 0.809 \\
Dry & $2 B \times 2 B$ & 0.150 & 0.467 & 9 & 9 & 57 & 12 & 56 & 0.91 & 0.749 \\
\hline
\end{tabular}


(d) Kishikawa (1970) also observed meiosis in rye plants grown experimentally under contrasting conditions of soil moisture and temperature, and again measured the efficiency of pairing and the number of micronuclei formed. The results of these experiments are summarised in table $4(a, b)$, the $\beta$ and $\lambda$ values being calculated in a simlar way to those above. He also made crosses between plants of the same $B$-class $(2 B)$, and from his data for the distribution of $B$ s among the progeny, using maximum likelihood and taking into account the mean $\beta$ and $\lambda$ values already calculated, estimates of $\mu$ and $\alpha$ have been made (table 4(c)).

Estimates of the parameters based on these experiments with Japanese rye enable us to confirm earlier suggestions (see Matthews and Jones, 1982) that environment can modify the transmission rates and population dynamics of the $B$ s by affecting their behaviour both at meiosis and pollen grain mitosis. At meiosis the non-pairing rate $\beta$ is increased both by a higher temperature and by a dry soil; the nonpaired loss rate $\lambda$ decreases with a rise in temperature and shows an increase under the dry conditions. The pollen grain mitosis parameters $\mu$ and $\alpha$, which relate to nondisjunction and direction rate respectively, both show a slight fall in magnitude with the higher temperature treatment, and this is reflected in the lower $B$-level in the progeny of $2.62 \mathrm{Bs} /$ plant compared with 3.05 at the low temperature-although part of this differences is also due to the higher meiotic loss under the high temperature regime. This supports Kishikawa's conclusion that the nondisjunction mechanism is sensitive to temperature. Lack of soil moisture has the effect of decreasing the direction rate, but the consequence to nondisjunction is minimal. A lower $B$-level of $2 \cdot 82 \mathrm{Bs} /$ plant is shown in the progeny grown in dry soil, compared to a level of 3.08 for those in moist soil, again reflecting the variation in parameters due to environment.

(e) Puertas et al., (1979) observed both meiotic metaphase and first pollen division anaphase in rye plants with differing cytoplasmic constitutions. From the data, using maximum likelihood, values of the four parameters $\beta, \lambda, \mu$ and $\alpha$ can be calculated (table 5). It can be seen from the metaphase data that there is actually an increase in $B$-level after meiosis, which is assumed to be due to sampling error. In any case there is probably very little loss occurring at this stage. The pollen grain mitosis data show that nondisjunction is far from complete (varying from $68-80$ per cent). However all the nondisjoined $B$ s appear to end up in the generative nucleus. Obviously the direction mechanism is very powerful in this case. In the same paper the authors present data to show that non-disjunction rate is also dependent to some extent on the age of the anther (as determined by the proportion of mononucleate to binucleate pollen). Nondisjunction takes place with a range of frequency from 55 per cent in older anthers to 81 per cent in the younger ones. Thus it seems that there are many factors controlling directed nondisjunction in rye and that even if environmental conditions are similar, its rate may vary greatly within the same anther at different stages of development. However for the purposes of simplicity in the present work, it is considered valid to use the mean directed 


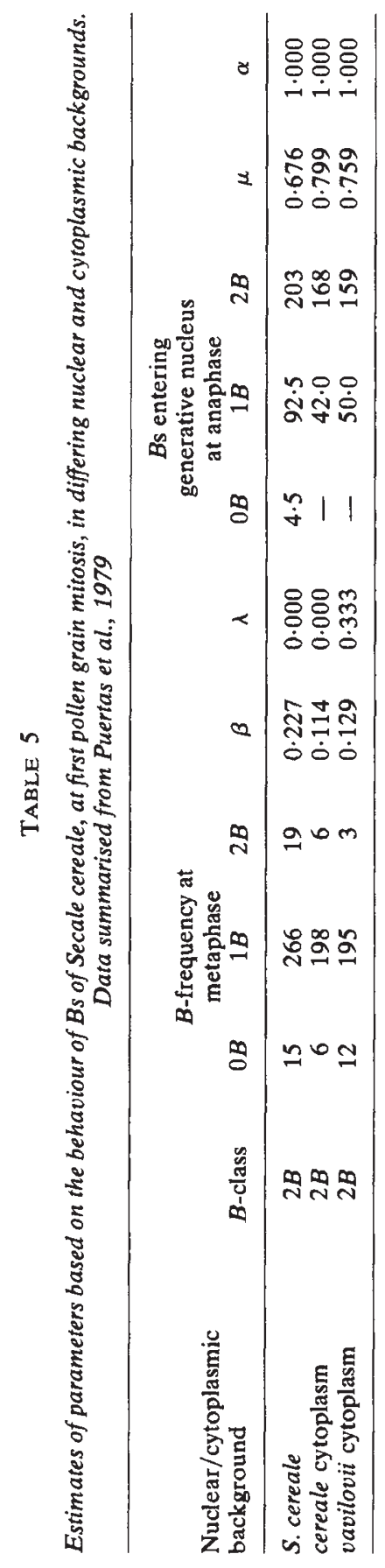




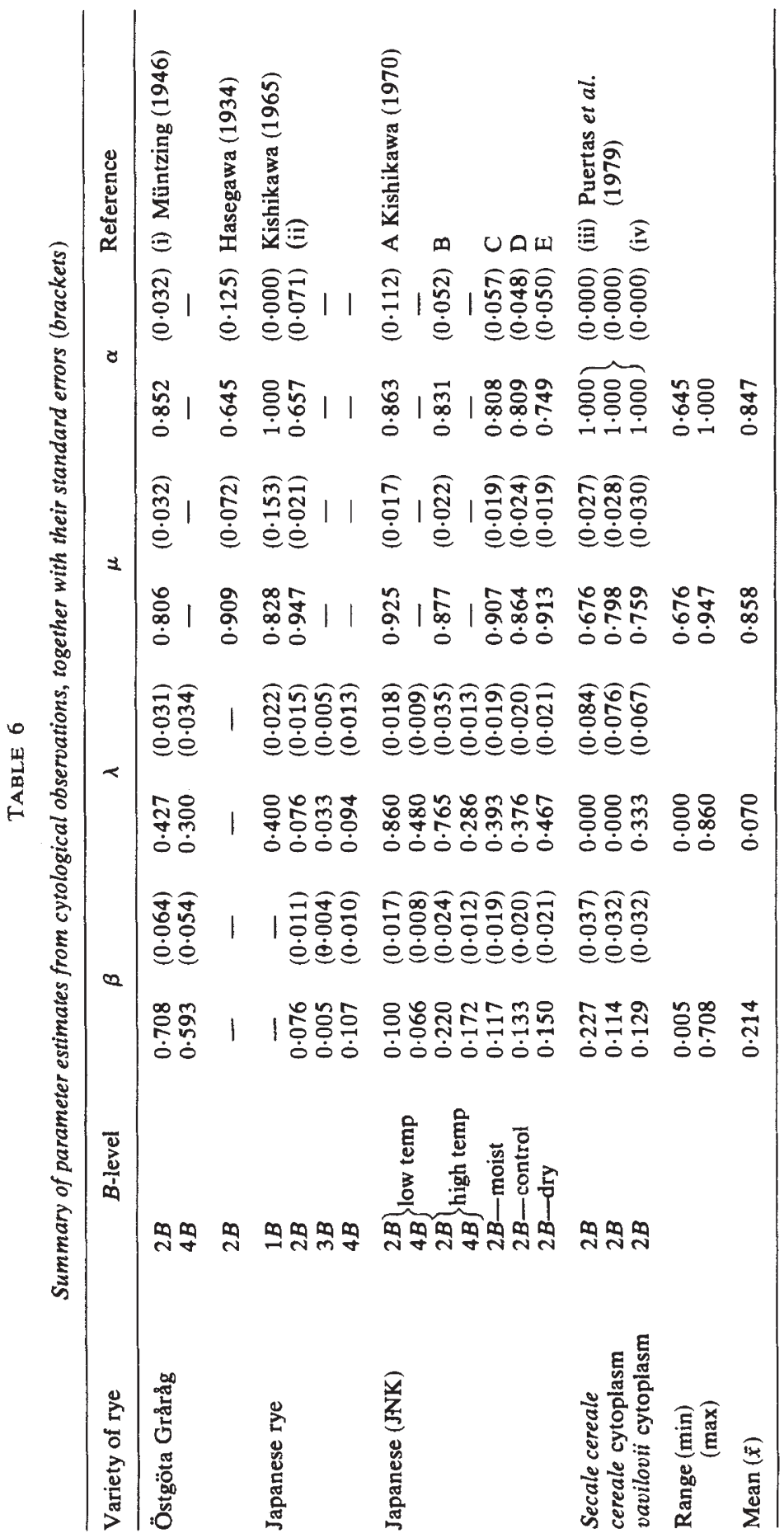


nondisjunction rate. There is some small variation in the parameters relating to differences in cytoplasmic background, but as Puertas et $a l$., point out it seems that within the cell the $B$ s are largely responsible for determining their own transmission characteristics.

We now have a range of values for each of the four parameters $\beta, \lambda$, $\mu$ and $\alpha$, calculated from cytological observations, which are summarised in table 6 . It can be seen that $\beta$ and $\lambda$ show the greatest variation with $\mu$ and $\alpha$ showing much less. The minor variation of $\mu$ may indicate that it is a relatively stable parameter, not being influenced much by either genotype or environment. Direction rate, on the other hand shows more variation and it is conceivable that differences in $B$-level between populations may be due to differing values for this parameter. The behaviour of $B$ s at meiosis is also obviously a major factor in the control of their level in populations, and it is quite likely that much of the variation is due to different rates of elimination (N.B. Östgöta Gråråg) at this stage.

\section{(ii) Estimates of gametic and plant weighting factors}

(a) Puertas and Carmona (1976) carried out a comparative study of the germination, and growth of tubes, in pollen of plants with and without $B$ chromosomes. Their results are summarised in table 7.

TABLE 7

Estimates of gametic weighting factors from germination and growth of pollen, from various $B$-classes of parent plant. Data from Puertas and Carmona, 1976

\begin{tabular}{lrrrrr}
\hline & \multicolumn{5}{c}{$B$-class of parent plants } \\
& $0 B$ & $1 B$ & $2 B$ & $3 B$ & $4 B$ \\
\hline Pollen grain germination (\%) & 30.7 & 43.0 & 48.0 & 20.3 & 18.9 \\
$\begin{array}{l}\text { Pollen tube growth (microns) } \\
\begin{array}{l}\text { 20 mins. after sowing } \\
40 \text { mins. after sowing }\end{array}\end{array}$ & 59.18 & 22.68 & 100.80 & - & 35.31 \\
$\begin{array}{l}\text { Gametic weighting factors } \\
\text { based on pollen germination }\end{array}$ & 114.90 & 70.77 & 169.99 & 10.29 & - \\
\hline
\end{tabular}

In general it appears that the $2 B$ pollen has a definite advantage over all the other classes, including that without any $B$ s at all, both in terms of germination percentage anc pollen tube growth. It is evident too that odd numbered combinaticns of $B$ s, have a disproportionately more harmful effect, on growth, and produce pollen grains which are vastly inferior in their growth potential-at least in vitro. A detailed account and discussion on the wider occurrence and significance of this odd-even effect is given in Jones and Rees (1982). The main difficulty with attempting to use these data for estimating gametic weighting factors, is that the actual $B$ chromosome classification of the pollen grains is unknown. We only know the $B$-class of plants giving rise to the pollen and the grains themselves will display some heterogeneity of $B$ numbers due to pairing and elimination effects of meiosis. As far as $0 B$ and $2 B$ plants are concered the situation is relatively straight forward: $O B$ plants will 
give exclusively $O B$ pollen and $2 B$ plants will give mainly pollen grains with $2 B \mathrm{~s}$ in their generative nuclei. This will occur because of regular distribution at meiosis when the $B$ s form a bivalent, and their subsequent doubling at first pollen mitosis. Plants with $1 B$ will give mainly $0 B$ and $2 B$ pollen grains, depending upon the degree of elimination of the unpaired univalents at meiosis. In $3 B$ and $4 B$ plants the situation will be less regular and higher numbers will occur. There is the confounding factor too of maternal influences over pollen grain development and physiology, which cannot be separated out, as well as the necessary assumptions we are making in equating pollen grains with gametes. In spite of these potential errors, and sources of unreliability, we have given the best estimates we can of gametic weighting based on the germination percentages in table 7. This avoids the complication of the odd-even effect found for pollen tube growth, and at a minimum the estimates give us some idea of the relative fitness of the different "gamete" $B$-classes. As was pointed out in the first paper in the series gametic weighting factor variation has in any case surprisingly little effect upon final equilibrium $B$ frequencies.

(b) Müntzing (1943) measured the effect of $B$ chromosomes on various aspects of vegetative and reproductive development in the Swedish rye variety Östgöta Gråråg. The results are given in summary form in table 8. As can be seen there is a gradual decrease in pollen fertility with increasing plant $\boldsymbol{B}$-level, as noted later by Puertas and Carmona (1976), and also a gradual decline in percentage seed set, which is a measure of female fertility. There appears to be an advantage conferred by the presence of a $1 B$ chromosome over all other classes, although this is based on very small numbers and may not be significant. However it does seem that on both the male and female side that small numbers of $B$ s have only a small, if any, detrimental effect on fertility, whereas large numbers reduce it severely. In a later publication Müntzing (1963) produced more extensive data on seed set which confirms the detrimental effects of high numbers of $B \mathrm{~s}$, and which shows as well that there is genetic variation for this character as between different strains of rye. This later paper also shows a slight enhancement of fertility due to $1 B$ in Östgöta Gråråg, but this is not the case for other varieties included in the study. Table 8 also includes counts on the number of seeds per ear, and the number of ears per plant, over two years. From these figures it has been possible to calculate the numbers of seeds per plant that each $B$-class of plant produces, and this then gives us an estimate of the plant weighting factors. Strictly the plant weighting factor is the relative reproductive potential of each $B$ class, which is the total number of seeds formed per zygote of the particular class. This takes into account plants dying during the growing period due to many factors from the environment. As we have no estimation of the number of plants that died in each class, it is impossible to calculate average numbers of seeds per zygote, but the numbers of seeds per plant should be reasonably close. The mean was taken for the two years $1941 / 42$ and values for the $4 B$ class and above combined into one (table 8 ). 


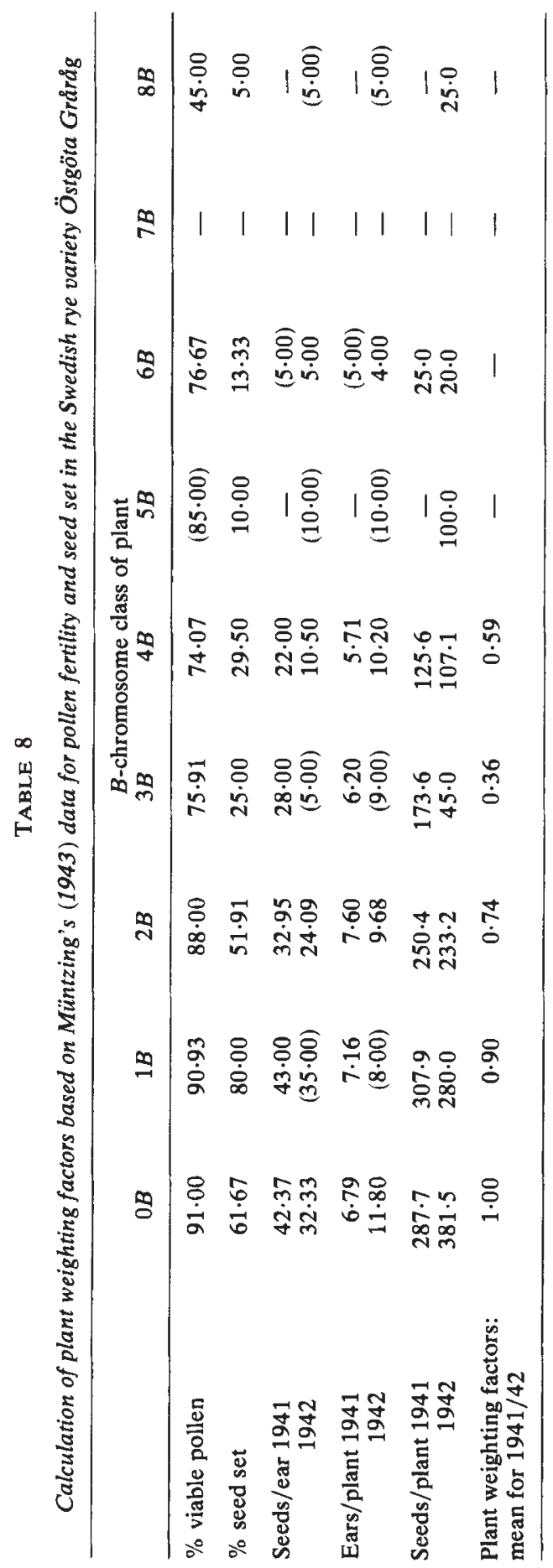




\section{(iii) Incorporation of literature estimates into the model}

The estimated real values of the parameters (table 6) have been incorporated into the model to see what kinds of simulations they produce. Four of the parameters, $\beta, \lambda, \mu$ and $\alpha$, have been estimated for a number of different varieties and experimental treatments, but the gametic and plant weighting parameters are represented only by single estimates, Puertas and Carmona (1976) and Müntzing (1943) respectively (table 7 and 8), and these have been used in all of the simulations. As these gametic and plant weighting factors play a relatively minor role anyway, in adjusting population $B$ frequencies (Matthews and Jones, 1982), we have had no hesitation in doing this and in having to restrict our comparisons to the more effective parameters involving meiosis and gametophyte development. For comparative purposes, as well as out of necessity, the parameter estimates used in

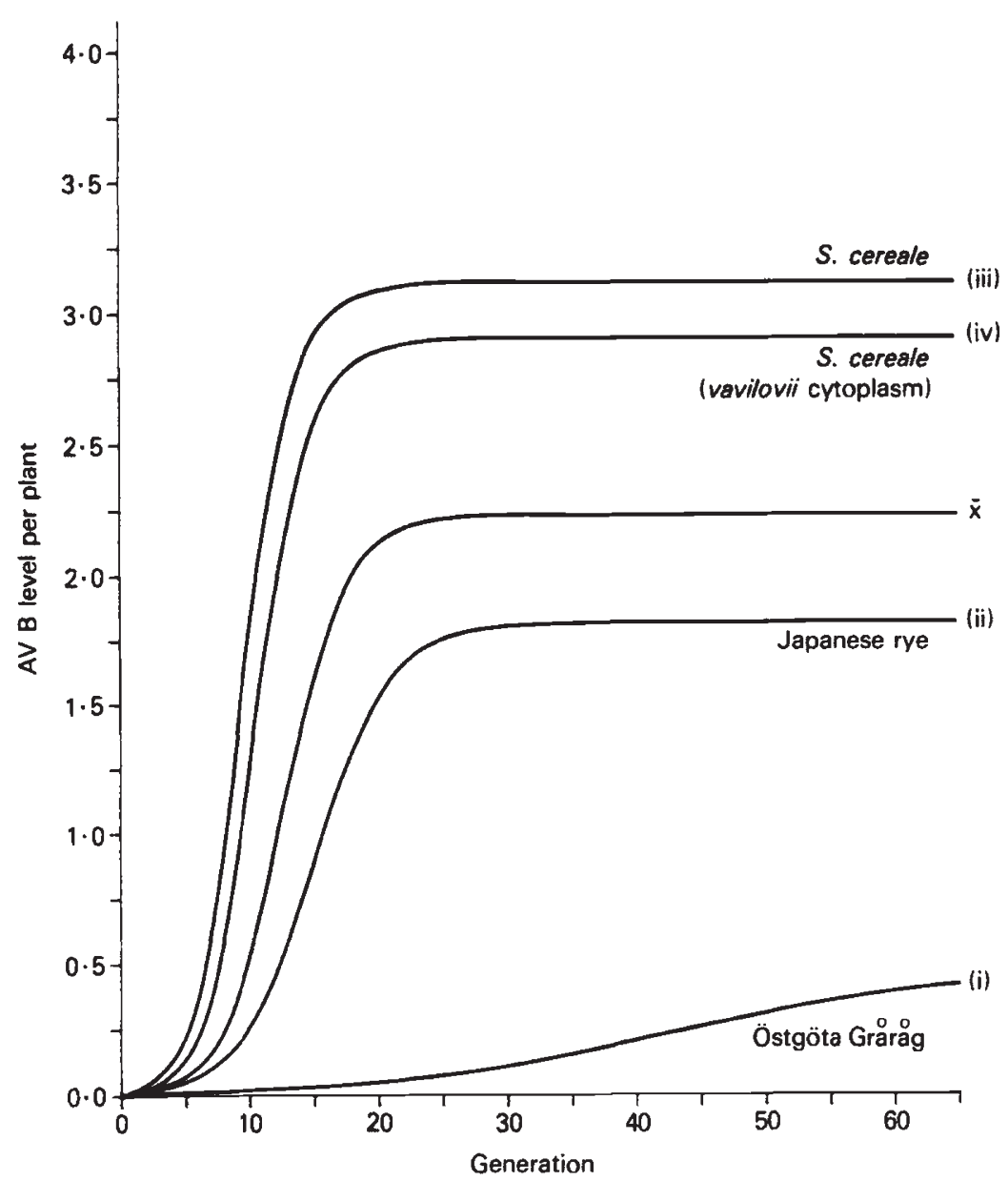

FIG. 1. Computer generated graph predicting the $B$ chromosome frequencies and accumulation characteristics of different experimental strains of rye, based on the estimated parameter values given in table 6 . Simulation $\bar{x}$ is from the mean of the estimated parameters of all strains and treatments. 
the simulations are based exclusively on the behaviour of the $2 B$ classes of plants, and it is appreciated that this restriction may also introduce some slight bias into the results. The simulations based on the parameter values given in table 6 are displayed in the computer generated graphs in figs. 1 and 2 .

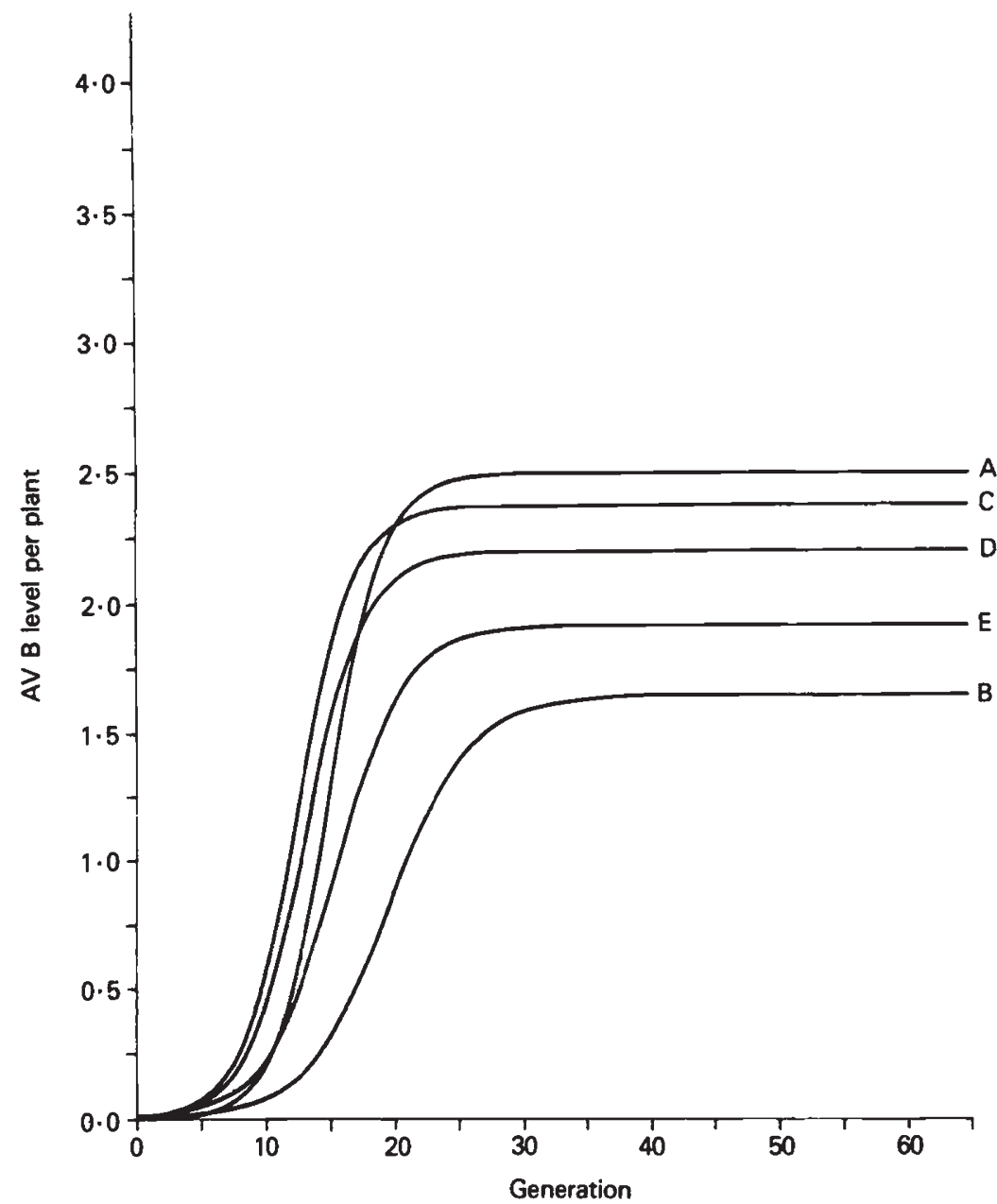

FIG. 2. Computer generated graph predicting the $B$ frequencies and accumulation characteristics in Japanese rye $(2 B)$, in response to different temperature and soil moisture conditions. Based on data of Kishikawa (1970), table 6. $A=$ low temp., $B=$ high temp., $C=$ moist, $\mathrm{D}=$ control for moisture treatment and $\mathrm{E}=\mathrm{dry}$.

Fig. 1 shows the patterns of accumulation and final equilibrium $B$ levels in three varieties of $S$. cereale, together with an overall mean $(\bar{x})$ based on all the parameter values given in table 6 . This overall mean includes the experimental treatments on Japanese rye by Kishikawa (1970), which are also presented separately in fig. 2 . 
The highest equilibrium levels shown in fig. 1 are those found for the variety used by Puertas et al., (1979), where slight differences are observed due to different cytoplasmic backgrounds into which the $B$ s were backcrossed. Simulation (iii) is based on the mean of two sets of parameters for $S$. cereale, and simulation (iv) for $S$. cereale $B$ s in a S. vavilovii cytoplasm (see table 6 and Puertas et al., 1979 for details). In both cases it will be noted that there is a minimum of meiotic elimination and that when nondisjunction does occur (at a rate of $c a .75$ per cent) all of the $B$ s are directed into the generative nucleus. The Japanese material studied by Kishikawa (1965), simulation (ii), stabilises at a lower level of about $1.75 \mathrm{~B} / \mathrm{plant}$, and this lower equilibrium is due mainly to a reduced direction rate of around 0.66 . In this variety too there is a minimum of meiotic elimination $(\beta=0.076, \lambda=0.076)$.

Östgöta Gråråg, the Swedish rye variety is known to have a very low natural $B$ frequency and a high rate of elimination of $B$ s at meiosis: in fact the original studies on this material all derive from a single $2 B$ population plant (Müntzing, 1943; Müntzing and Prakken, 1941). The model is in agreement in showing a very low equilibrium $B$ level for this variety (fig. 1 (i)) as well as a rate of accumulation which is barely perceptible over the initial generations. The drive mechanism itself is as effective in this material as it is in any of the other varieties, but it is balanced by the high rate of meiotic elimination $(\beta=0.708, \lambda=0.427)$.

The simulations in fig. 2 result from using the estimated parameter values derived from the data of Kishikawa, 1970. In the experiment on environmentally induced variation in $B$ chromosome transmission Kishikawa eliminated genetic differences by working with clonally propagated tillers of the natural JNK strain of Japanese rye. High and low temperature treatments were applied during meiosis and reproduction, as well as three different levels of soil water-the control treatment was intermediate between moist and dry. Kishikawa found significant differences between treatments in respect of the average number of $B$ s per plant among progeny of controlled crosses, and also showed that the low and high levels of transmission corresponded to low and high rates of pairing and of elimination of unpaired $B \mathrm{~s}$, as well as to some effects on seed fertility. In all crosses the number of $B$ s per progeny plant exceeded that of the parents.

On the basis of the parameter values obtained from Kishikawa the graphs in fig. 2 again predict a rapidly accumulating $B$ frequency, rising to their equilibrium values between the fifteenth and thirtieth generation. The equilibria shown in the graphs correspond, in their order of magnitude, with the parent-offspring transmission rates. The largest difference found, 3.07 and $2.62 \mathrm{Bs} /$ plant for the progeny of $2 B \times 2 B$ crosses in the low and high temperature regimes respectively, give the largest difference in equilibrium levels, graphs A and B respectively, in fig. 2. It is notable too that the parameter most affected by these environmental variables is the nonpairing rate $(\beta)$ and that the directed nondisjunction parameters $(\mu$ and $\alpha)$ are relatively unaffected (table 6 ). Changes in moisture level are less effective than temperature in influencing equilibrium $B$ levels, but again the graphs correspond with the experiment in that the order of treatments $C>D>E$ in fig. 2 fit with the $2 B \times 2 B$ crossing results of $3.08,2.95$ and $2.82 \mathrm{Bs} /$ plant for progeny in the moist, control and dry treatments respectively. 


\section{Discusion}

The results from estimates of real parameter values given in this paper confirm the conclusions drawn from the earlier theoretical model. As far as we can see the drive mechanism based on directed nondisjunction in the gametophytes, is universally operative at a high rate ( $\mu$ and $\alpha$ table 6 ), and as a consequence the $B$ s inevitably accumulate within populations. Adjustment of equilibrium levels is effectively brought about by variation in the nonpairing rate parameter $\beta$ and the nonpaired loss rate parameter $\lambda$, rather than through fluctuations in $\mu$ and $\alpha$ : these two latter parameters remain remarkably stable over a wide range of genetic and environmental conditions. As yet we have been unable to gain any really satisfactory estimates of the values of gametic and plant weighting factors, and this is an area of inquiry identified for further study and investigation.

Varietal differences suggest genetically controlled sources of variation, either between the $B s$ of different strains or else in the interactions between the $B \mathrm{~s}$ and their respective $A$ chromosome complements, although we cannot discriminate between these sources on the present evidence. In the experiments reported here there is no standardisation of environments within which to make varietal comparisons. The most we can say at present, as was pointed out in the first paper, is that there are known structural variants among the standard $B$ s of rye, relating to populations of different origin and habitat, and that when some of these diverse types are brought together within the same individual plant their divergence is confirmed by reduced levels of pairing and chiasma formation at meiosis (Müntzing, 1951). It is known too that the $B s$, in rye, as in maize (Carlson, 1978; Carlson and Chou, 1981; Ward, 1973), have a high level of autonomy in the control over thier own behaviour and inheritance, independently of the $A$ chromosome background genotype. When the $B$ s of rye are placed as addition lines into hexaploid wheat for instance, and separated from their own $A$ complement, they are still able to undergo directed nondisjunction at the appropriate time and place, in the gametophytes, as is witnessed by an increase in their numbers among the progenies. Their meiotic pairing, however, is very poor in the alien background (Müntzing et al., 1969), and it would appear that the meiotic parameters $\beta$ and $\lambda$ are more sensitive to background genotype than are the gametophyte parameters $\mu$ and $\alpha$ (see also table 5). These seems good reason to believe, therefore, that part of the variation at least in $B$ chromosome transmission characteristics, between different strains and populations of rye, can be attributed to the independently controlled activities of the $B$ s themselves. It is also interesting to note, in relation to the selfish aspect of $B \mathrm{~s}$, that when the "Chinese Spring" variety of wheat is pollinated by $B$ chromosome rye, the pollen of $2 B$ plants has a competitive advantage over that from plants without $B$ s (Zeven and Keijer, 1980).

The evidence for fluctuations in $B$ frequencies, and in population equilibrium levels, due to environmental effects, is more straight-forward. It is shown in the case of Kishikawa's experiment described above, where the main influence is on meiotic parameters, and it comes too from other sources. Müntzing (1967), for example, sampled a primitive population of rye from Transbaikal in 1952, and subsequently maintained the population 
in a field plot at Lund (in Sweden) over 14 generations of open pollination. During that period the $B$ frequency declined from an initial 28 per cent plants with $B$ s down to 10 per cent; although there is no information on which of the parameter values were modified.

In some other species, notably Myrmeleottix maculatus (Barker, 1966; Hewitt and Ruscoe, 1971; Hewitt and Brown, 1970; Hewitt and John, 1970; Ramel, 1980) and Pseudococcus obscurus (Nur, 1969) the evidence for environmental involvment in regulating $B$ frequencies is just as firmly established, as was mentioned in paper 1 (see also Jones, 1983 and Jones and Rees, 1982, for further references and discussions on this point). Environment can be expected to have influence too in so far as it is likely to modify the variation in reproductive and developmental fitness induced by Bs. Nur (1969) and Kimura and Kayano (1961), have drawn attention to this point in relation to the range of stable equilibrium $B$ frequencies which exist in populations of the mealy bug Pseudococcus obscurus and of Lilium callosum respectively. It could be for this reason that in many of the better known examples of $B$ chromosome polymorphism that the $B$ s are found in their highest frequencies under conditions which are optimum for the species to thrive (e.g., Podisma pedestris, Hewitt, 1975; Lilium auratum, Ogihara, 1962; Trandescantia edwardsiana, Brown, 1960; Centaurea scabiosa, Fröst, 1958; Festuca pratensis, Bosemark, 1956; Phleum phleoides, Bosemark, 1967; Myrmeleotettix maculatus, Barker, 1966; Hewitt and Ruscoe, 1971; Ramel, 1980). If we accept that in general terms $B$ s are harmful, rather than beneficial, to the individuals and species that carry them, then their accumulation and spread within a population is likely to be more successful under conditions where the species itself is thriving than where it is struggling for existence at the margins of its range of distribution.

Confining our remarks to those species with a drive mechanism for accumulation of $B \mathrm{~s}$, which we discussed in paper 1, the polymorphisms appears to be influenced by three main factors. There is (i) the accumulation mechanism itself which is primarily responsible for disseminating the $B \mathrm{~s}$ throughout populations, and this is balanced by (ii) instabilities in the inheritance of $B$ s which leads to their elimination at mitosis and/or meiosis together with (iii) the adverse effects which the Bs have upon physiological and reproductive fitness. Equilibrium levels are brought about by the balance between these factors and by their variable expression in given environments.

Returning to the question of genetic variation between $B \mathrm{~s}$, and their relationships with $A$ s, we must at least consider the possiblity of some long term co-evolution between the two, and surmise that there may be a parallel here with the gene-for-gene relationships which exist between host crop plants and their fungal pathogens, and that this parallel could well serve as a basis on which to extend our future investigations. There is no doubt that as far as rye is concerned the $B$ s must have a strategy for survival which involves on their part an optimum, rather than a maximum, of accumulation potential. An over aggressive $B$ would not achieve an equilibrium -it would quickly rise to a mean level of $4 B$ /plant which would then lead to severe sterility and extermination of the population. 


\section{REFERENCES}

BARKER, J. F. 1966. Climatological distribution of a grasshopper supernumerary chromosome. Evolution, 20, 665-667.

BOSEMARK, N. O. 1956. On accessory chromosomes in Festuca pratensis. III. Frequency and geographical distribution of plants with accessory chromosomes. Hereditas, 42, 189-210.

BOSEMARK, N. O. 1967. Edaphic factors and the geographical distribution of accessory chromosomes, in Phleum phleoides. Hereditas, 57, 239-262.

BROWN, W. V. 1960. Supernumerary chromosomes in a population of Tradescantia edwardsiana. The Southwestern Naturalist, 5, 49-60.

CARLSON, w. R. 1978. The $B$ chromosome of corn. Ann. Rev. Genet., 16, 5-23.

CARLSON, W. R. AND CHOU, T. S. 1981. B chromosome nondisjunction in corn: control by factors near the centromere. Genetics, 97, 379-389.

FRÖST, S, 1958. The geographical distribution of accessory chromosomes in Centaurea scabiosa. Hereditas, 44, 75-111.

HASEGAWA, N. 1934. A cytological study of 8-chromosome rye. Cytologia, 6, 68-77.

HEWITT, G. M. 1975. A sex-chromosome hybrid zone in the grasshopper Podisma pedestris (Orthoptera: Acrididae). Heredity, 35, 375-387.

HEWITT, G. M. AND BROWN, F. M. 1970. The $B$ chromosome system of Myrmeleotettix maculatus. V. A steep cline in East Anglia. Heredity, 25, 363-371.

HEWITT, G. M. AND JOHN, B. 1970. The B chromosome system of Myrmeleottix maculatus (Thunb.) IV. The dynamics. Evolution, 24, 169-180.

HEWITT, G. M. AND RUSCOE, C. 1971. Changes in microclimate correlated with a cline for $B$-chromosomes in the grasshopper Myrmeleotettix maculatus (Thunb.) (Orthoptera: Acrididae). J. Animal Ecology, 40, 753-765.

JONES, R. N. 1983. Are $B$ chromosomes selfish? In Cavalier-Smith, T. (ed.) DNA and Evolution: Natural Selection and Genome Size, John Wiley and Sons Ltd.

JONES, R. N. AND REES, H. 1982. B Chromosomes. Academic Press.

KIMURA, M. AND KAYANO, H. 1961. The maintenance of supernumerary chromosomes in wild populations of Lilium callosum by preferential segregation. Genetics, 46, 1699-1712.

KishikAwA, H. 1965. Cytogenetic studies of $B$ chromosomes in rye, Secale cereale L., in Japan. Agric. Bull. Saga Univ., 21, 1-81.

KISHIKAWA, H. 1970. Effects of temperature and soil moisture on frequency of accessory chromosomes in rye, Secale cereale L. Jap. J. Breeding, 20, 269-274.

MATHER, K. 1963. The Measurement of Linkage in Heredity. Methuen and Co. Ltd., London.

MATHER, K. 1972. Statistical Analysis in Biology. Science Paperback, Chapman and Hall Ltd., London.

MATTHEWS, R. B. 1981. Studies on B Chromosomes with Particular References to Plant Disease. Ph.D. Thesis, University of Wales.

MATTHEWS, R. B. AND JONES, R. N. 1982. Dynamics of the $B$ chromosome polymorphism in rye. I. Simulated populations. Heredity, 48, 345-369.

MÜNTZING, A. 1943. Genetical effects of duplicated fragment chromosomes in rye. Hereditas, 29, 91-112.

MÜNTZING, A. 1946. Cytological studies of extra fragment chromosomes in rye. III. The mechanism of non-disjunction at the pollen mitosis. Hereditas, 32, 97-119.

MÜNTZING, A. 1951. The meiotic pairing of iso-chromosomes in rye. Portugaliae Acta Biologica Series A. Goldschmidt Vol., 831-860.

MÜNTZING, A. 1963. Effects of accessory chromosomes in diploid and tetraploid rye. Hereditas, 49, 371-426.

MÜNTZING, A. 1967. Some main results from investigations of accessory chromosomes. Hereditas, 57, 432-438.

MÜNTZING, A. AND PRAKKEN, R. 1941. Chromosomal aberrations in rye populations. Hereditas, 27, 273-308.

MÜNTZING, A., JAWORSKA, H. AND CARLIBOM, C. 1969. Studies of meiosis in the Lindström strain of wheat carrying accessory chromosomes of rye. Hereditas, 61, 179-207.

NUR, U. 1969. Harmful $B$ chromosomes in a mealy bug: additional evidence. Chromosoma, $28,280-297$.

OGIHARA, R. 1962. B chromosomes of Lilium auratum LINL. II. Frequency and distribution pattern of plants with the $B$ chromosomes in natural populations. La Kromosomo, 53-54, $1785-1793$.

PUERTAS, M. J. AND CARMONA, R. 1976. Greater ability of pollen tube growth in rye plants with $2 B$ chromosomes. Theor. Appl. Genet., 47, 41-43. 
PUERTAS, M. J., DIEZ, M. AND CARMONA, R. 1979. Rye B chromosome behaviour at first and second pollen mitosis and its relationship with anther maturity. Theor. Appl. Genet., 54, 65-68.

RAMEL, C. 1980. A B chromosome system of Myrmeleotettix maculatus (Thun.) (Orthoptera: Acrididae) in Sweden. Hereditas, 92, 309-313.

WARD, E. J. 1973. Nondisjunction: localisation of the controlling site in the maize $B$ chromosome. Genetics, 73, 387-391.

ZEVEN, A. AND KEIJER, C. J. 1980. The effect of the number of $B$ chromosomes in rye on its crossability with wheat. Cereal Res. Communications, 8, 491-494. 